\title{
An Efficient 3-D FDTD Model of Electromagnetic Wave Propagation in Magnetized Plasma
}

\author{
Alireza Samimi, Member, IEEE, and Jamesina J. Simpson, Senior Member, IEEE
}

\begin{abstract}
Modeling electromagnetic wave propagation in the upper atmosphere is important for space weather effects, satellite communications ionospheric modification experiments, and many other applications. We propose a new methodology for solving and incorporating the current equation into the finite-difference time-domain (FDTD) form of Maxwell's equations for modeling electromagnetic wave propagation in magnetized plasma. This approach employs a version of Boris's algorithm applied to particle-in-cell plasma computational models. There are four primary advantages of this new method over previously developed three-dimensional FDTD models of electromagnetic wave propagation in magnetized plasma. Specifically, it: 1) requires less memory; 2) is more than $50 \%$ faster; 3 ) is easier to implement; and 4) permits the use of two different time step increments when solving the current equation versus Maxwell's equations that is useful for modeling high collisional regimes. The new algorithm is faster because it solves all the equations explicitly and there is no need to solve complicated matrix equations. Modeling of higher altitude ranges and higher frequency electromagnetic waves is much more feasible using this new method. Results of the new FDTD magnetized plasma model are provided and validated.
\end{abstract}

Index Terms-Earth, electromagnetic (EM) wave propagation, finite-difference time-domain (FDTD) method, ionosphere, magnetized cold plasma.

\section{INTRODUCTION}

$\mathbf{E}$ LECTROMAGNETIC (EM) wave propagation in the upper atmosphere and magnetosphere is critically important for investigations of space weather hazards, satellite communications, radar, remote-sensing, geophysics (such as propagation from lightning [1]), and for ionospheric modification experiments. The ionospheric modification experiment is a relatively new technique for exploring the upper atmosphere [2]-[4]. For instance, a strong, high-frequency (HF) EM wave transmitted to the ionosphere from the ground can modify the ionosphere and excite new EM waves in the ionosphere called stimulated electromagnetic emission (SEEs) [5]-[10]. The SEE spectral features provide diagnostic information about the ionosphere (e.g., [11]).

Manuscript received March 05, 2014; revised August 10, 2014; accepted October 22, 2014. Date of publication October 31, 2014; date of current version December 31, 2014. This work was supported by National Science Foundation CAREER Award Grant 0955404.

The authors are with the Department of Electrical and Computer Engineering, University of Utah, Salt Lake City, UT 84112 USA (e-mail: ar.samimi@utah. edu; jamesina.simpson@utah.edu).

Color versions of one or more of the figures in this paper are available online at http://ieeexplore.iee.org.

Digital Object Identifier 10.1109/TAP.2014.2366203
The full-vector Maxwell's equations finite-difference timedomain (FDTD) method [12] has previously been applied to simulate electromagnetic wave propagation in the Earth-ionosphere waveguide. The advantages of using FDTD for ionospheric wave propagation include:

- As a grid-based method it may:

- Include 3-D spatial material variations of the ionosphere composition, topography/bathymetry, lithosphere composition, geomagnetic field, targets, antennas, etc.

- Calculate the complex shielding, shadowing, scattering, and diffraction of those waves upon reaching the Earth's surface/other obstacles.

- Calculate electromagnetic propagation on a local or global scale.

- Account for any number of simultaneous sources (antennas, plane waves, lightning strikes, etc.)

- Permit any number of observation points, and the ability to create movies of the propagating waves

- As a time-domain method it may:

- Model arbitrary time-varying source waveforms, movement of objects, time variations in the ionosphere

- Provide propagation results over a large spectral bandwidth via a discrete Fourier transform

- By accounting for magnetized ionospheric plasma physics it may:

- Calculate all important ionospheric effects on signals, including absorption, refraction, phase and group delay, frequency shift, polarization, and Faraday rotation.

Initial FDTD modeling of EM wave propagation in the Earth ionosphere waveguide were localized and two-dimensional [13]-[17]. As powerful supercomputing resources have become more available, fully 3-D global models have been developed (e.g., [18]-[20]). Simpson and Taflove [21] and Simpson [22] provide a comprehensive review of the FDTD models developed for investigation of wave propagation in the Earth-ionosphere waveguide.

For simplicity and to reduce computational requirements, global FDTD models initially assumed an isotropic conductivity profile for the ionosphere. This was sufficient for ultra low-, extremely low-, and very low-frequency (ULF, ELF, and VLF) wave propagation [23]. Applications of global FDTD models assuming an isotropic ionosphere ranged from remote sensing of ionospheric anomalies [24] and underground oil fields [25], [26] to modeling of Schuman resonances [20], hypothetical ELF earthquake precursors [27], and space weather effects on the operation of power grids [28].

Modeling of higher frequency wave propagation or propagation at higher altitudes, however, requires consideration of an 
ionospheric medium that is magnetized and anisotropic. In order to account for the effect of the geomagnetic field and anisotropic ionospheric conductivity on wave propagation in the upper atmosphere, the global FDTD model of Simpson and Taflove, [19] has been advanced to solve not only Maxwell's equations but also the simplified momentum equation in the ionosphere [29], [30]. To accomplish this, the local, 2-D cylindrical FDTD algorithm of [1] was extended to 3-D Cartesian [29] and then to a global FDTD grid [30]. The 3-D global FDTD model of Yu and Simpson [30] may directly model Faraday rotation, which is the rotation of the plane of polarization of a linearly polarized wave propagating through a magnetized medium.

Unfortunately, to account for the magnetized plasma physics, additional field components and coefficients must be calculated and stored which makes the 3-D algorithm memory intensive [29], [30]. It also is difficult and slow to implement because of the resulting matrix equations and inverse matrix calculations. Finally, the algorithm is unstable for high collision frequencies unless the time step is reduced. This means that there are still many ionospheric EM wave propagation simulation scenarios that are still infeasible to model using existing FDTD modeling techniques. In [31], Bérenger proposes a new 2-D FDTD magnetized plasma scheme that is based on semi-exponential differencing. In this method, the impact of the collision frequency is considered analytically. This method reduces the memory requirements relative to previous methods; however, it is still implicit and requires the solution of matrix equations. Furthermore, it is mentioned that semi-exponential differencing yields accurate results for ELF-VLF propagation [31]. It is not clear whether this method can be applied to other frequency regimes as well.

In this paper, a new method for solving the current equation is proposed that significantly increases the computational speed and reduces the memory requirements. Implementation of this new algorithm is similar to the simple classic FDTD model [12] and all equations are solved explicitly. The proposed technique borrows ideas widely implemented in particle in cell (PIC) simulations for plasma. Relative to the algorithm of [29], [30], the proposed algorithm:

1) Requires storage of three additional components $\left(J_{x}, J_{y}, J_{z}\right)$ per grid cell versus nine (two levels of storage in time for the $\vec{J}$ components and a second level of storage of the $\vec{E}$ components). It also avoids the need to either store or re-calculate a coefficient matrix of at least size $6 \times 6$ at every grid cell:

2) is more than $50 \%$ faster because no matrix equation is required to be solved and all equations are explicit;

3) different time step increments may be used for Maxwell's equations versus the current equation which is helpful for modeling high collisional regimes;

4) it is easier to implement.

This paper is organized as follows. In the next section, the new algorithm is explained; then, empirical stability criteria are discussed; afterward, several validation experiments are performed; next, the new algorithm is compared with the previous 3-D anisotropic model [29], [30]; the paper then ends with conclusions and an acknowledgement.

\section{MEthoD}

For simplicity, the algorithm is initially presented in Cartesian coordinates. The proposed method is a modified version of the algorithm developed by Hu and Cummer [1] and applied to [29]. It solves the simplified momentum equation to find the electric current in the ionosphere, or in more general terms, in any magnetized plasma medium. What is unique about the proposed approach is that it utilizes modeling techniques from Borris [32], which is widely used in well-established particle-in-cell (PIC) plasma computational method [33]

The presumption of the method is that the density of the particle species in the ionosphere is known. It also assumes that the temporal variation of the density in comparison to the temporal variation of the particle velocity is negligible, i.e., the plasma is cold and no thermal pressure is considered. Therefore, the momentum equation for each species is simplified to

$$
\frac{\partial \overrightarrow{J_{j}}}{\partial t}+\nu_{j} \overrightarrow{J_{j}}=\epsilon_{0} \omega_{p j}^{2} \vec{E}-\overrightarrow{\omega_{c j}} \times \overrightarrow{J_{j}}
$$

where $\overrightarrow{J_{j}}$ is electric current due to j species, where the subscript $j$ represents electron or ion species, $\nu_{j}$ is the collision frequency, $\epsilon_{0}$ is the electric permittivity, $\omega_{p j}$ is the $j$ species plasma angular frequency, and $\overrightarrow{\omega_{c j}}$ is the $j$ species angular gyro-frequency. Note that the angular gyro-frequency is a scalar value determined by $\omega_{c j}=q_{j} B_{0} / m_{j}(\mathrm{rad} / \mathrm{s})$, where $q_{j}$ is the $j$ species charge, $B_{0}$ is the background magnetic field and $m_{j}$ is the $j$ species mass. In this paper, the vector of the $j$ species angular gyro-frequency is defined as $\overrightarrow{\omega_{c j}}=\left(q_{j} B_{0} / m_{j}\right) \overrightarrow{B_{0}} /\left|\overrightarrow{B_{0}}\right|$, which is along the magnetic field for positive ions and is anti-parallel to the magnetic field for electrons and negative ions.

Equation (1) is incorporated into Maxwell's equations as

$$
\begin{aligned}
& \nabla \times \vec{H}=\epsilon_{0} \frac{\partial \vec{E}}{\partial t}+\sum \overrightarrow{J_{j}}+\overrightarrow{J_{s}} \\
& \nabla \times \vec{E}=\mu_{0} \frac{\partial \vec{H}}{\partial t}
\end{aligned}
$$

where $\overrightarrow{J_{s}}$ is the external source current density. The discretization technique that is used is based on the Yee algorithm where the transverse magnetic (TM) and the transverse electric (TE) planes are stacked in the $x$-, $y$ - and $z$-directions. The $\vec{H}$ field components are calculated at each half time steps, i.e., $(n+1 / 2)$ and the $\vec{E}$ fields at every integer time step, i.e., $(n)$. It is assumed that the reader is familiar with the classic FDTD method and the Yee algorithm [12], so discretization of Maxwell's equations is not repeated here. The FDTD form of Maxwell's equations for the Earth-ionosphere model are described in [19]. Thus, the focus of this section is on the efficient computational solution of (1) and its incorporation into (2) ((3) is not modified relative to traditional FDTD).

It appears from (2) that the electric current density components should be collocated with the electric field components. However, in Yee's FDTD algorithm, components of the electric field are not collocated. Here, it will be shown that in order to efficiently solve (1), all the current density components are required to be at the same spatial position. To resolve this issue, 
spatial averaging will be used as needed to calculate current density components at positions at which they are not located.

There are two terms in (1) that makes the calculations burdensome: the collision term and the cross-product term. The cross-product term represents rotation of the particles around the geomagnetic field. Solving the cross-product term will be described in Section II-A below. Consideration of the collision term will be reserved for Section II-B.

\section{A. Collision-Less Plasma}

In the absence of collisions, (1) may be solved using an algorithm introduced by Borris [32] for calculating the velocity of particles in particle-in-cell (PIC) plasma computations [33]. The cross-product term in (1), as mentioned before, represents rotation around the magnetic field. Suppose there is no collision and the electric field is zero, $\left(\left(\partial \overrightarrow{J_{j}}\right) /(\partial t)=-\overrightarrow{\omega_{c j}} \times \overrightarrow{J_{j}}\right)$. Via a dot multiplication of this equation with the current density vector, it may be readily shown that the amplitude of the current density vector remains constant $\left(\left(\partial \overrightarrow{\left|J_{j}\right|^{2}}\right) /(\partial t)=0\right)$. This is the basis of the Boris algorithm. In the absence of collisions, (1) may be written in discrete form as follows:

$\frac{\overrightarrow{J_{j}^{n+1 / 2}}-\overrightarrow{J_{j}^{n-1 / 2}}}{\Delta t}=\epsilon_{0} \omega_{p e}^{2} \overrightarrow{E^{n}}-\overrightarrow{\omega_{c j}} \times\left(\frac{\overrightarrow{J_{j}^{n+1 / 2}}+\overrightarrow{J_{j}^{n-1 / 2}}}{2}\right)$

where $n$ is the time step number. As was explained earlier in this section and can be seen from (4), the electric current density vector components should be collocated with the electric field components. However, in the time domain, the current density vectors are calculated at the same time as the $\vec{H}$ field components, i.e., at each half time steps $(n+1 / 2)$. In order to simplify (4), the $\vec{E}$ field should be incorporated into the current density vector term. By defining two auxiliary current density vectors as follows:

$$
\begin{aligned}
& \overrightarrow{J^{+}}=\overrightarrow{J_{j}^{n+1 / 2}}-\frac{\Delta t \epsilon_{0} \omega_{p e}^{2} \overrightarrow{E^{n}}}{2} \\
& \overrightarrow{J^{-}}=\overrightarrow{J_{j}^{n-1 / 2}}+\frac{\Delta t \epsilon_{0} \omega_{p e}^{2} \overrightarrow{E^{n}}}{2} .
\end{aligned}
$$

Equation (4) is then simplified to

$$
\frac{\overrightarrow{J^{+}}-\overrightarrow{J^{-}}}{\Delta t}=-\overrightarrow{\omega_{c j}} \times\left(\frac{\overrightarrow{J^{+}}+\overrightarrow{J^{-}}}{2}\right)
$$

The cross-product does not change the amplitude of the current density vector, i.e., $\left|\overrightarrow{J^{+}}\right|=\left|\overrightarrow{J^{-}}\right|$. However, the direction of the vector is changed. Fig. 1 demonstrates the rotation of the current density vector around $\overrightarrow{\omega_{c j}}$, which, for simplicity only in the figure, is assumed to be perpendicular to the current components. In Fig. 1, the direction of the B-field is into the paper.

Note that the addition $\left(\overrightarrow{J^{+}}+\overrightarrow{J^{-}}\right)$and subtraction $\left(\overrightarrow{J^{+}}-\right.$ $\overrightarrow{J^{-}}$) vectors (shown in blue in Fig. 1) are in fact diagonals of a

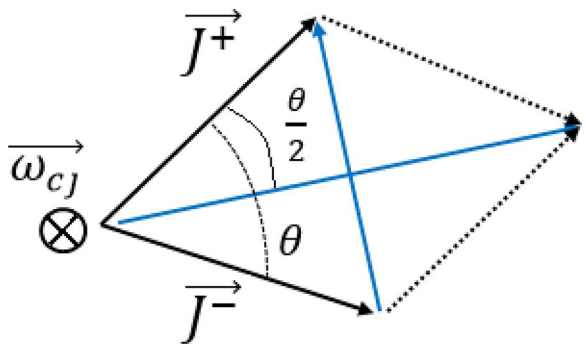

Fig. 1. Rotation of current density vector around $\vec{\omega}_{c j}$. Note that only for the simplicity of displaying, $\vec{\omega}_{c j}$ is assumed to be perpendicular to the current density vector and toward the paper. Note that for electrons and negative ions direction of $\vec{\omega}_{c j}$ and consequently direction of rotation are reversed. Figure adapted from [33].

rhombus. The diagonals of a rhombus are perpendicular to one another and cut each other in half. Thus, from Fig. 1, the angle of rotation is

$$
\frac{\theta}{2}=\tan ^{-1} \frac{\left|\overrightarrow{J^{+}}-\overrightarrow{J^{-}}\right|}{\left|\overrightarrow{J^{+}}+\overrightarrow{J^{-}}\right|}
$$

and from (7)

$$
\frac{\left|\overrightarrow{J^{+}}-\overrightarrow{J^{-}}\right|}{\left|\overrightarrow{J^{+}}+\overrightarrow{J^{-}}\right|}=\frac{\left|\overrightarrow{\omega_{c j}}\right| \Delta t}{2}
$$

therefore, the angle of rotation obtains from the following equation:

$$
\frac{\theta}{2}=\tan ^{-1} \frac{\left|\overrightarrow{J^{+}}-\overrightarrow{J^{-}}\right|}{\left|\overrightarrow{J^{+}}+\overrightarrow{J^{-}}\right|}=\tan ^{-1} \frac{\left|\overrightarrow{\omega_{c j}}\right| \Delta t}{2} .
$$

It will be shown that the rotation angle should always be $\theta \leq$ $115^{\circ}$. The $\overrightarrow{J^{+}}$may be found in four steps as follows [32], [33]:

$$
\begin{aligned}
& \overrightarrow{J_{0}}=\overrightarrow{J^{-}} \times \vec{t} \\
& \overrightarrow{J_{1}}=\overrightarrow{J^{-}}+\overrightarrow{J_{0}} \\
& \overrightarrow{J_{2}}=\overrightarrow{J_{1}} \times \vec{s} \\
& \overrightarrow{J^{+}}=\overrightarrow{J^{-}}+\overrightarrow{J_{2}}
\end{aligned}
$$

where $\vec{t} \quad=\quad\left(\overrightarrow{\omega_{c j}}\right) /\left(\left|\overrightarrow{\omega_{c j}}\right|\right) \tan (\theta) /(2) \quad$ and $\vec{s}=\left(\overrightarrow{\omega_{c j}}\right) /\left(\left|\overrightarrow{\omega_{c j}}\right|\right) \sin \theta$. It should be emphasized again that for electrons and negative ions the gyro-frequency $\overrightarrow{\omega_{c j}}$ is negative (the gyro-frequency vector is anti-parallel to the background magnetic field). To summarize, the steps of the algorithm are as follows: 1) First, the $x, y, z$ components of the $\overrightarrow{J_{j}^{n-1 / 2}}$ and $\overrightarrow{E^{n}}$ are used in (6) to calculate the $x, y, z$ components of $\overrightarrow{J^{-}}$. 2) Then, $\overrightarrow{J^{-}}$is written as a vector and is incorporated into (11a)-(11d). 3) Next, the $x, y, z$ components of $\overrightarrow{J^{+}}$are used in (5) to find the new time-step values of $\overrightarrow{J_{j}^{n+1 / 2}}$.4) Finally, the new $\overrightarrow{J_{j}^{n+1 / 2}}$ values are incorporated into (2) for the next iteration cycle. Thus, only (2), (3), (5), (6), and (11) are actually implemented in this scheme. 


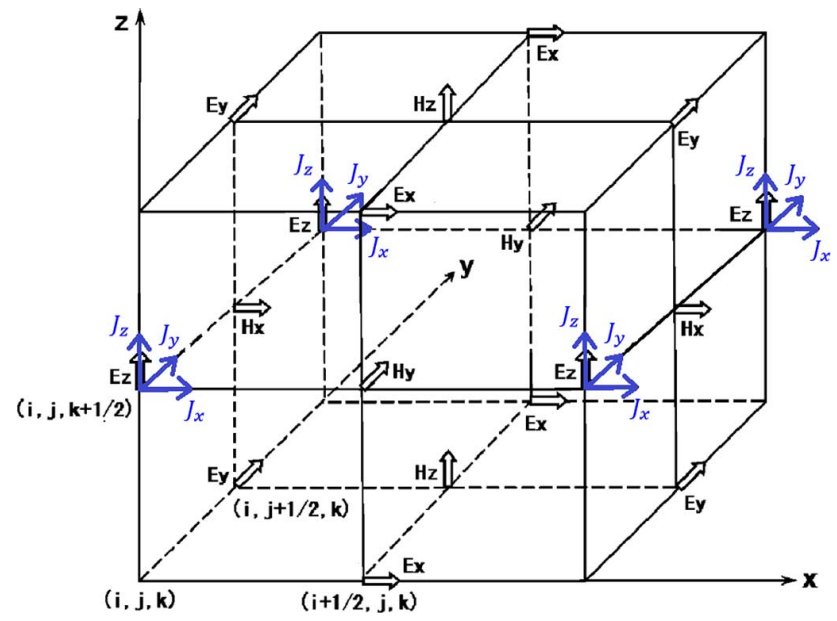

Fig. 2. Yee cell illustrating the spatial positioning of the magnetized plasma field components.

This algorithm finds all three, collocated components of the current density vector. In the Yee discretization scheme, the position of each electric field component is different. In the magnetized plasma algorithm, current density vector components are required at each position of the electric field components in order to solve Ampere's law [(2)]. On the other hand, all the electric field components are required at the grid points wherein the current equation is solved. It could be possible to solve the current equation at all grid points where the electric field components are located, but this would be computationally very expensive. Another solution is to use the average of the neighboring current densities in (2) and the average of the neighboring electric field components in (1).

The current density vectors could be calculated at any of the grid points where an electric field component is located. We arbitrarily choose to solve (collocate) all the current density vector components at the position where the $z$ component of the $\vec{E}$ field is located. Fig. 2 illustrates the positions of the $\vec{H}$ fields, $\vec{E}$ fields and $\vec{J}$ vector components in the Yee cell. Note that the subscript $j$ of the current vectors ( $j$ represents electrons or ions species) not included for simplicity in Fig. 2.

In order to solve (1) (the current equation), all electric field components at the $E_{z}$ location are required. Thus $x$ and $y$ components of the electric field are averaged as follows:

$$
\begin{gathered}
E_{x,(i, j, k+1 / 2)}=\left[E_{x,(i+1 / 2, j, k)}+E_{x,(i-1 / 2, j, k)}\right. \\
\left.\quad+E_{x,(i+1 / 2, j, k+1)}+E_{x,(i-1 / 2, j, k+1)}\right] / 4 \\
E_{y,(i, j, k+1 / 2)}=\left[E_{y,(i, j+1 / 2, k)}+E_{y,(i, j-1 / 2, k)}\right. \\
\left.\quad+E_{y,(i, j+1 / 2, k+1)}+E_{y,(i, j-1 / 2, k+1)}\right] / 4 .
\end{gathered}
$$

To solve (2) (Ampere's law equation), $\overrightarrow{J_{j x}}\left(J_{j y}\right)$ should be known at $E_{x}\left(E_{y}\right)$ position. Similar to (12), (13)), the current density vectors are averaged

$$
\begin{gathered}
J_{j x,(i+1 / 2, j, k)}=\left[J_{j x,(i, j, k+1 / 2)}+J_{j x,(i+1, j, k+1 / 2)}\right. \\
\left.\quad+J_{j x,(i, j, k-1 / 2)}+J_{j x,(i+1, j, k-1 / 2)}\right] / 4 \\
J_{j y,(i, j+1 / 2, k)}=\left[J_{j y,(i, j, k+1 / 2)}+J_{j y,(i, j+1, k+1 / 2)}\right. \\
\left.\quad+J_{j y,(i, j, k-1 / 2)}+J_{j y,(i, j+1, k-1 / 2)}\right] / 4 .
\end{gathered}
$$

\section{B. Collisional Regime}

The difficulty in solving (1) in the collisional regimes is that the current density vector is needed at time step $n+1 / 2$, which is not yet known. In order to solve this issue, a two-step method known as predictor-corrector is used here. In the first step, the current density vector at $n-1 / 2$ is used to predict the current density vector at $n+1 / 2$. Then the predicted current density vector from the first (predictor) step is used in the second (corrector) step and all the equations are solved again. The second, new current vector found at $n+1 / 2$ is known as the corrector current density vector. The average of the predicted current density vector and the corrector current density vector at $n+1 / 2$ is used as the final value for the current density vector at $n+1 / 2$. The predictor-corrector method also known as the MacCormack method is second-order accurate [35], [36].

The discrete form of (1) in the predictor step is as follows:

$$
\begin{aligned}
& \frac{\overrightarrow{J_{j, p}^{n+1 / 2}}-\overrightarrow{J_{j}^{n-1 / 2}}}{\Delta t}+\nu \overrightarrow{J_{j}^{n-1 / 2}} \\
& \quad=\epsilon_{0} \omega_{p e}^{2} \overrightarrow{E^{n}}-\overrightarrow{\omega_{c j}} \times\left(\frac{\overrightarrow{J_{j, p}^{n+1 / 2}}+\overrightarrow{J_{j}^{n-1 / 2}}}{2}\right) .
\end{aligned}
$$

The auxiliary current density vectors are defined as

$$
\begin{aligned}
& \overrightarrow{J^{+}}=\overrightarrow{J_{j, p}^{n+1 / 2}}-\frac{\Delta t \epsilon_{0} \omega_{p e}^{2} \overrightarrow{E^{n}}}{2}+\frac{\Delta t \nu \overrightarrow{J_{j}^{n-1 / 2}}}{2} \\
& \overrightarrow{J^{-}}=\overrightarrow{J_{j}^{n-1 / 2}}+\frac{\Delta t \epsilon_{0} \omega_{p e}^{2} \overrightarrow{E^{n}}}{2}-\frac{\Delta t \nu \overrightarrow{J_{j}^{n-1 / 2}}}{2} .
\end{aligned}
$$

The remaining procedure is analogous to what was discussed in Section II-A. In the corrector step, (1) is discretized as follows:

$$
\begin{aligned}
& \frac{\overrightarrow{J_{j, c}^{n+1 / 2}}-\overrightarrow{J_{j}^{n-1 / 2}}}{\Delta t}+\nu \overrightarrow{J_{j, p}^{n+1 / 2}} \\
& \quad=\epsilon_{0} \omega_{p e}^{2} \overrightarrow{E^{n}}-\overrightarrow{\omega_{c j}} \times\left(\frac{\overrightarrow{J_{j, c}^{n+1 / 2}}+\overrightarrow{J_{j}^{n-1 / 2}}}{2}\right) .
\end{aligned}
$$

The auxiliary current density vectors are defined as

$$
\begin{aligned}
& \overrightarrow{J^{+}}=\overrightarrow{J_{j, c}^{n+1 / 2}}-\frac{\Delta t \epsilon_{0} \omega_{p e}^{2} \overrightarrow{E^{n}}}{2}+\frac{\Delta t \nu \overrightarrow{J_{j, p}^{n+1 / 2}}}{2} \\
& \overrightarrow{J^{-}}=\overrightarrow{J_{j}^{n-1 / 2}}+\frac{\Delta t \epsilon_{0} \omega_{p e}^{2} \overrightarrow{E^{n}}}{2}-\frac{\Delta t \nu \overrightarrow{J_{j, p}^{n+1 / 2}}}{2} .
\end{aligned}
$$

The final current density vector is

$$
\overrightarrow{J_{j}^{n+1 / 2}}=\frac{\overrightarrow{J_{j, p}^{n+1 / 2}}+\overrightarrow{J_{j, c}^{n+1 / 2}}}{2} .
$$

\section{Adaptation to the Global FDTD Model}

Before providing validation results, it is necessary to briefly explain the incorporation of the discrete current density equation into the global FDTD Earth-ionosphere model [19]. In the 


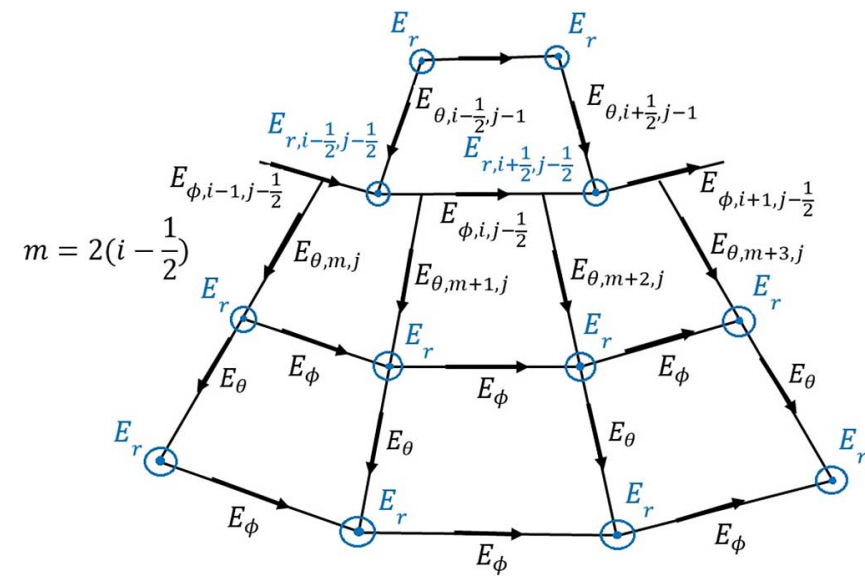

Fig. 3. Distribution of electric field components at the merging cells in the global Earth-ionosphere model. Note that $E_{r}$ components are half a grid cell above the $E_{\theta}, E_{\phi}$ components.

Equatorial Regions, the grid cells are nearly cubical. In this case, the $(x, y, z)$ orientations are replaced with $(\phi, \theta, r)$ and the procedure of Sections II-A and II-B is analogous in the global FDTD model as for a Cartesian mesh, but near the polar region, since grid cells in the $\phi-$ (east-west) direction are merged to avoid small time step values, incorporation of the current equation into (2) is a bit more tedious. Fig. 3 shows merging cells near the North Pole region. Only $\vec{E}$ field vector components are displayed in the figure. The $E_{r}$ components shown in blue and the $E_{\theta}, E_{\phi}$ components displayed in black are stacked (offset) in the $r$ direction. The $E_{r}$ components are half a grid size $(\Delta r / 2)$ above the $E_{\theta}, E_{\phi}$ components. At the merging cells, the number of $E_{\phi}$ components around the $E_{r}$ components is similar to the non-merging cell. However, the number of $E_{\theta}$ components is doubled on the side farther away from the North Pole; thus, in order to find the $E_{\theta}$ at the $E_{r}$ location, (13) should be modified as follows:

$$
\begin{aligned}
E_{\theta,(i-1 / 2, j-1 / 2, k+1 / 2)} & \\
= & {\left[\frac{E_{\theta,(m, j+1, k)}+E_{\theta,(m+1, j+1, k)}}{2}\right.} \\
& +\frac{E_{\theta,(m, j+1, k+1)}+E_{\theta,(m+1, j+1, k+1)}}{2} \\
& \left.+E_{\theta,(i, j-1, k)}+E_{\theta,(i, j-1, k+1)}\right] / 4 .
\end{aligned}
$$

At the merging of cells, (15) also should be modified. Since all the current density components are collocated with $E_{r}$, from Fig. 3, the modified equation can be derived as

$$
\begin{aligned}
J_{j \theta,(} & m+1, j, k) \\
= & {\left[\frac{3 J_{j \theta,(i-1 / 2, j-1 / 2, k-1 / 2)}}{4}+\frac{J_{j \theta,(i+1 / 2, j-1 / 2, k-1 / 2)}}{4}\right.} \\
& +\frac{3 J_{j \theta,(i-1 / 2, j-1 / 2, k+1 / 2)}}{4}+\frac{J_{j \theta,(i+1 / 2, j-1 / 2, k+1 / 2)}}{4} \\
& \left.+J_{j \theta,(m+1, j+1 / 2, k-1 / 2)}+J_{j \theta,(m+1, j+1 / 2, k+1 / 2)}\right] / 4
\end{aligned}
$$

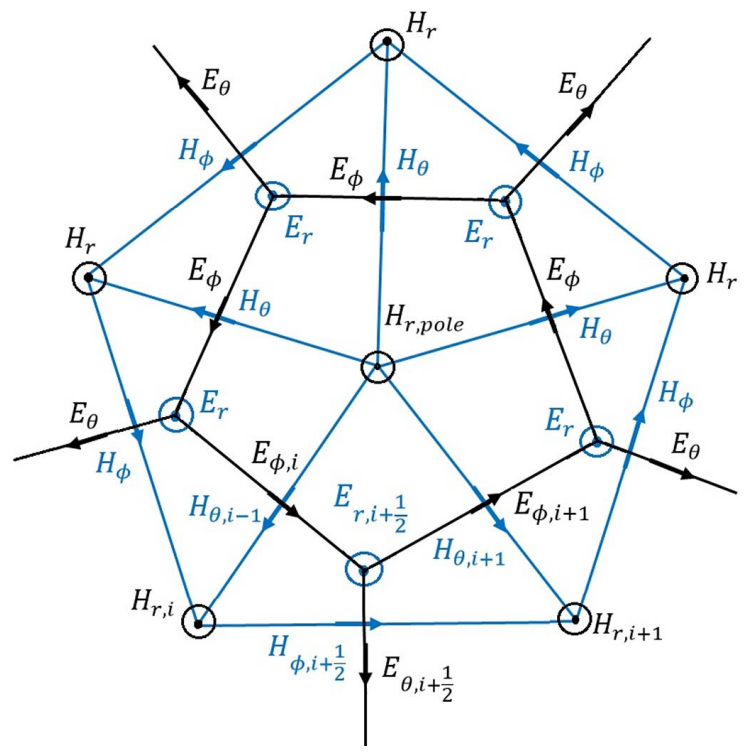

Fig. 4. Grid cell distribution at the North Pole in the global Earth-ionosphere model. Note that $E_{r}$ components are half a grid cell above the $E_{\theta}, E_{\phi}$ components.

$$
\begin{aligned}
J_{j \theta,(} & m+2, j, k) \\
= & {\left[\frac{J_{j \theta,(i-1 / 2, j-1 / 2, k-1 / 2)}}{4}+\frac{3 J_{j \theta,(i+1 / 2, j-1 / 2, k-1 / 2)}}{4}\right.} \\
& +\frac{J_{j \theta,(i-1 / 2, j-1 / 2, k+1 / 2)}}{4}+\frac{3 J_{j \theta,(i+1 / 2, j-1 / 2, k+1 / 2)}}{4} \\
& \left.+J_{j \theta,(m+2, j+1 / 2, k-1 / 2)}+J_{j \theta,(m+2, j+1 / 2, k+1 / 2)}\right] / 4 .
\end{aligned}
$$

Finally, Fig. 4 shows grid cells around and at the North Pole. It is obvious that $E_{\theta}$ components only exist on one side of the $E_{r}$ component; thus, (13) near the North Pole for non-merging cell takes the following form:

$$
E_{\theta,(i-1 / 2, j-1 / 2, k+1 / 2)}=\frac{E_{\theta(i, j+1, k)}+E_{\theta,(i, j+1, k+1)}}{2} .
$$

If at the North pole, grid cells are merged (which is not shown in Fig. 4), the average of the $E_{\theta}$ should be calculated as follows:

$$
\begin{aligned}
& E_{\theta,(i-1 / 2, j-1 / 2, k+1 / 2)} \\
& =\left(\frac{E_{\theta,(m, j+1, k)}+E_{\theta,(m+1, j+1, k)}}{2}\right. \\
& \left.\quad+\frac{E_{\theta,(m, j+1, k+1)}+E_{\theta,(m+1, j+1, k+1)}}{2}\right) / 2 .
\end{aligned}
$$

\section{STABILITY CONDITION}

A complete theoretical stability analysis of Maxwell's equations coupled with the simplified momentum equation is challenging and is beyond the scope of this paper. Instead, we conduct a number of tests, to determine the stability condition empirically. To accurately solve the current density equation, two criteria should be met:

1) According to the Nyquist sampling theorem, the sampling frequency should be at least twice the highest frequency component of the signal that is sampled [34]. Therefore, $\Delta t_{c}<(1) /\left(2 f_{c e}\right)=(\pi) /\left(\omega_{c e}\right)$ which results in $\theta \leq$ 
$115^{\circ}$. Smaller angles will achieve more accurate results. A comparison of the time step value, angle of rotation and electron cyclotron frequency is provided in Section IV. A typical electron gyro-frequency in the ionosphere is approximately $\omega_{c e}=2 \pi\left(1.4 \times 10^{6}\right) \mathrm{rad} / \mathrm{s}$, and the corresponding time step value for solving the current equation would be $\Delta t_{c}<3.57 \times 10^{-7} \mathrm{~s}$.

2) The second condition is determined by the collision frequency: $\nu \Delta t_{c}<0.1$.

It should be noted that these criteria for choosing the time step value is only applied to the current equation solver. Also, it should be emphasized these conditions hold even for the previous anisotropic model [29], [30] (which does not provide the flexibility to choose two different time steps for some modeling scenarios).

On the other hand, numerical examinations of the stability condition of the coupled equations show that for calculating the $\vec{E}$ and $\vec{H}$ fields, the maximum time step value for which the algorithm is stable should meet two criteria:

1) the Courant stability condition, i.e., $\Delta t$

$$
\text { (1) } /\left(c \sqrt{1 / \Delta x^{2}+1 / \Delta y^{2}+1 / \Delta z^{2}}\right)[12]
$$

2) $\sqrt{1-\left(\omega_{p e} \Delta t / 2\right)^{2}}>0.9$.

The latter criterion is vaguely mentioned by $\mathrm{Hu}$ and Cummer [1] for other explicit methods as well. In the method presented here, if $\Delta t<\Delta t_{c}$, then the whole set of equations utilizes $\Delta t$. However, if $\Delta t_{c}<\Delta t$, it is possible to use two different time steps for solving Maxwell's equation versus the current equation. In such cases, in order to accommodate this inconsistency in the time step values, for every time step that the $\vec{E}$ and $\vec{H}$ fields are calculated, the current equation solver should be repeated such that the total passed time of both equations become equal. Note that for all of the iterations of the current equation solver having a smaller time step value, the electric field at time step $n$ is used. In other words, $\vec{E}$ between $n-1 / 2$ and $n+1 / 2$ is assumed to be constant and equal to the electric field at $n$, i.e., $\left.\vec{E}\right|^{n}$.

In the $\mathrm{D}$ region of the ionosphere, the plasma density is relatively low and the collision frequency is high. The previous anisotropic model [29], [30] is not able to consider the D region unless the (one) time step value (for the current equation and Maxwell's equations) is reduced, but reducing the time step value results in a very computationally inefficient algorithm. The new algorithm suggested here overcomes this restriction by allowing two different time step values for the calculation of the current equation and Maxwell's equations. Obviously, the time step restriction that is enforced by the plasma frequency can in some modeling cases be smaller than the maximum allowed time step in the previous anisotropic model [29], [30]. However, the new model still requires much less memory and is faster than the previous model. A detailed comparison of the two methods is provided in Section V.

\section{VALIDATIONS}

\section{A. Current Equation Solution}

1) Test 1: Before combining the Boris algorithm, predictorcorrector method, and current equation with FDTD Maxwell's equations, the current equation was solved independently for different electric field intensities and collision frequencies while using a typical geomagnetic field strength. The Boris algorithm is based on the physical interpretation of the current equation, and it is built on the fact that the amplitude of the auxiliary current density vector does not change. In this test, the updated current density vector $\left(J_{e}^{n+1 / 2}\right)$ is incorporated back into the discrete form of (1) to check how accurately the current vector is updated for different electric field intensities, collision frequencies and time step values. The other goal of this test is to examine the performance of the combined Boris algorithm and predictor-corrector method. The error was defined as the difference between the right-hand side and the left-hand side of the equation:

$$
\begin{aligned}
\text { error } & =\left(\overrightarrow{J_{e}^{n+1 / 2}}-\overrightarrow{J_{e}^{n-1 / 2}}\right)+\nu \Delta t\left(\frac{\overrightarrow{J_{e}^{n+1 / 2}}+\overrightarrow{J_{e}^{n-1 / 2}}}{2}\right) \\
& -\Delta t \epsilon_{0} \omega_{p e}^{2} \overrightarrow{E^{n}}-\Delta t \overrightarrow{\omega_{c e}} \times\left(\frac{\overrightarrow{J_{e}^{n+1 / 2}}+\overrightarrow{J_{e}^{n-1 / 2}}}{2}\right) .
\end{aligned}
$$

An initial value is assigned to the current density vector at $n-1 / 2$, and the electric field at $n$; then (1) is solved one million time steps (the current density vector was updated one million times) and the maximum and minimum error described by (28) is recorded. This test was performed for many arbitrary electric field intensities, electric current densities, collision frequencies and time step values. Table I shows some examples of the parameter regimes that were chosen for this test and the corresponding absolute error value.

For the magnetic field strength, a typical value of the geomagnetic field intensity at around $100 \mathrm{~km}$ altitude is chosen according to the International Geomagnetic Reference Field (IGRF) model. We note that the geomagnetic field strength reduces as the altitude increases. The maximum allowed time step value is determined by the maximum electron gyro-frequency $\left(\Delta t_{c}<\pi / \omega_{c e}\right)$. Since $100 \mathrm{~km}$ altitude is almost bottom of the ionosphere and the corresponding geomagnetic field strength is the largest there, this magnetic field strength is chosen for the test.

In all the collisionless cases, the error is negligible (order of $10^{-6}$ ). For stronger electric fields, the error increases but it is still on the order of $10^{-6}$, which is extremely small. Note that the electric field intensities and current densities that are used in this test are quite large. These field intensities were only chosen for checking the robustness of the method. The error increases slightly for larger time step values. It may be inferred from Table I that increasing the current density to the order of $10^{3}$ has almost no effect on the minimum and maximum error.

In the collisional regime, the error is larger than for the collisionless cases and this can make the code unstable. Thus, the collision frequency is another factor that restricts the maximum allowed time step values. It is important to mention that in the collisional regime the error is not cumulative. The maximum error is obtained at the first iteration and the minimum error is achieved at the last iteration except for cases wherein the code becomes unstable. It is stated in Section III that the empirical criterion to avoid an unstable current density vector while solving 
TABLE I

MaXimum and Minimum Computational Errors Obtained From (21) After One Million Iterations of Updating the CuRRent Density Vector In (1). Note That the Listed Electric Density Vector Is the Initial Value Used at the First Time SteP

\begin{tabular}{|c|c|c|c|c|c|c|}
\hline$\left(B_{x}, B_{y}, B_{z}\right)(n T)$ & $\left(J_{x}, J_{y}, J_{z}\right)(A / m)$ & $\left(E_{x}, E_{y}, E_{z}\right)(V / m)$ & $\nu(1 / s)$ & $\Delta t(s)$ & $\mid$ error $\left.\right|_{\min }$ & $\mid$ error $\left.\right|_{\max }$ \\
\hline$(4.7,0.03,2.6) \times 10^{4}$ & $(1,1,1)$ & $(0,0,0)$ & 0 & $12 \times 10^{-8}$ & 0 & $0.8 \times 10^{-15}$ \\
\hline$(4.7,0.03,2.6) \times 10^{4}$ & $(1,1,1)$ & $(1,1,1) \times 10^{5}$ & 0 & $12 \times 10^{-8}$ & $1.6 \times 10^{-16}$ & $1.4 \times 10^{-10}$ \\
\hline$(4.7,0.03,2.6) \times 10^{4}$ & $(1,1,1)$ & $(1,300,7000) \times 10^{5}$ & 0 & $12 \times 10^{-8}$ & $2.3 \times 10^{-13}$ & $4.5 \times 10^{-7}$ \\
\hline$(4.7,0.03,2.6) \times 10^{4}$ & $(10,6,2) \times 10^{3}$ & $(1,300,7000) \times 10^{5}$ & 0 & $12 \times 10^{-8}$ & $1.42 \times 10^{-14}$ & $3.13 \times 10^{-9}$ \\
\hline$(4.7,0.03,2.6) \times 10^{4}$ & $(10,6,2) \times 10^{3}$ & $(1,300,7000) \times 10^{5}$ & 0 & $12 \times 10^{-8}$ & $1.0 \times 10^{-12}$ & $4.3 \times 10^{-7}$ \\
\hline$(4.7,0.03,2.6) \times 10^{4}$ & $(10,6,2) \times 10^{3}$ & $(1,300,7000) \times 10^{5}$ & 0 & $3 \times 10^{-7}$ & $1.6 \times 10^{-12}$ & $2.0 \times 10^{-6}$ \\
\hline$(4.7,0.03,2.6) \times 10^{4}$ & $(1,1,1)$ & $(0,0,0)$ & $4.9 \times 10^{5}$ & $12 \times 10^{-8}$ & 0 & $1.8 \times 10^{-2}$ \\
\hline$(4.7,0.03,2.6) \times 10^{4}$ & $(1,1,1)$ & $(1,1,1) \times 10^{5}$ & $4.9 \times 10^{5}$ & $12 \times 10^{-8}$ & $1.8 \times 10^{-16}$ & $1.9 \times 10^{-2}$ \\
\hline$(4.7,0.03,2.6) \times 10^{4}$ & $(1,1,1)$ & $(1,300,7000) \times 10^{5}$ & $4.9 \times 10^{5}$ & $12 \times 10^{-8}$ & $7.3 \times 10^{-13}$ & 37.4 \\
\hline$(4.7,0.03,2.6) \times 10^{4}$ & $(10,6,2) \times 10^{3}$ & $(1,300,7000) \times 10^{5}$ & $4.9 \times 10^{5}$ & $6 \times 10^{-8}$ & $4.14 \times 10$ & 2.33 \\
\hline$(4.7,0.03,2.6) \times 10^{4}$ & $(10,6,2) \times 10^{3}$ & $(1,300,7000) \times 10^{5}$ & $4.9 \times 10^{5}$ & $12 \times 10^{-8}$ & $1.2 \times 10^{-12}$ & 145.8 \\
\hline$(4.7,0.03,2.6) \times 10^{4}$ & $(10,6,2) \times 10^{3}$ & $(1,300,7000) \times 10^{5}$ & $4.9 \times 10^{5}$ & $3 \times 10^{-7}$ & Unstable & Unstable \\
\hline
\end{tabular}

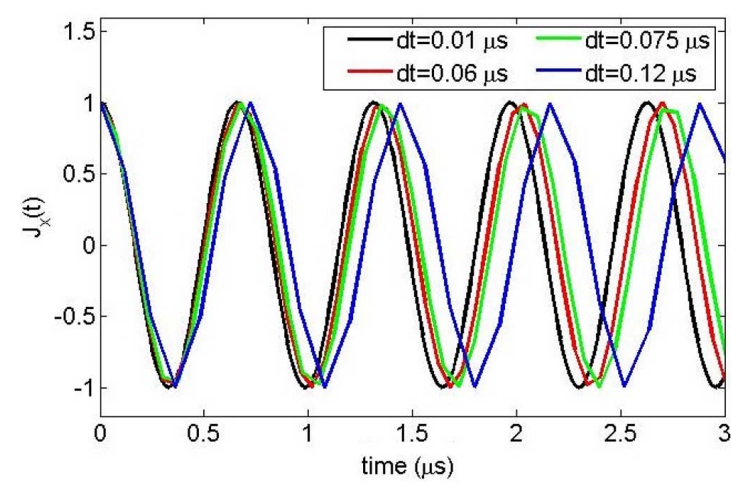

Fig. 5. Oscillation of the current density vector perpendicular to the background magnetic field. As the time step value increases the numerical electron cyclotron frequency decreases.

the current equation in the collisional regime is $\nu t<0.1$. In addition to the maximum and minimum error, the amplitude of the current density vector was checked in all the iterations. For the cases wherein the electric field is zero and there is no collision, the amplitude of the current density vector remains constant, which represents conservation of the energy that was mentioned previously. The electric field enhances the current density amplitude and the collision reduces its amplitude, which is consistent with the physics of the equation.

2) Test 2: In the absence of the electric field and collision, the analytical solution of the current equation is a sinusoidal current density vector that represents rotation around the magnetic field. The frequency of oscillation is the gyro-frequency of the corresponding species. For instance, it is easy to show that for a magnetic field in the $z$ direction, the current density vector due to electrons is

$$
\overrightarrow{J_{e}}=\left|\overrightarrow{J_{e}}\right| \cos \left(\left|\overrightarrow{\omega_{c e}}\right| t\right) \hat{a}_{x}+\left|\overrightarrow{J_{e}}\right| \sin \left(\left|\overrightarrow{\omega_{c e}}\right| t\right) \hat{a}_{y}
$$

As a second test of Boris algorithm, an initial current density vector due to electrons is assumed to be in the $x$ direction, i.e., $J_{x}(0)=1 \mathrm{~A} / \mathrm{m}^{2}$, and the magnetic field in the $\mathrm{z}$ direction. The magnetic field strength is $B_{z}=54418.4 \mathrm{nT}$, which corresponds to $\omega_{c e}=9.55 \times 10^{6}(\mathrm{rad} / \mathrm{s})$. The current equation is solved for different time step values and numerical oscillation frequencies are compared to the electron gyro-frequency. Fig. 5 shows the $x$-component of the electric current density vector. Table II shows the rotation angle per time step obtained
TABLE II

COMPUTATIONAL ERROR OF THE ElECTRON CyCLOTRON FREQUENCY

\begin{tabular}{cccc}
$\Delta t(s)$ & $\theta($ degree $)$ & $\omega_{c e N}(\mathrm{rad} / \mathrm{s})$ & $\frac{\omega_{c e}-\omega_{c N}}{\omega_{c e}}$ \\
\hline $1 \times 10^{-8}$ & $5.4^{\circ}$ & $9.52 \times 10^{6}$ & 0.005 \\
$6 \times 10^{-8}$ & $32^{\circ}$ & $9.52 \times 10^{6}$ & 0.005 \\
$7.5 \times 10^{-8}$ & $39.4^{\circ}$ & $9.37 \times 10^{6}$ & 0.01 \\
$1.2 \times 10^{-7}$ & $59.7^{\circ}$ & $8.72 \times 10^{6}$ & 0.08 \\
$3 \times 10^{-7}$ & $110.6^{\circ}$ & $6.98 \times 10^{6}$ & 0.27 \\
$3.27 \times 10^{-7}$ & $115^{\circ}$ & $6.4 \times 10^{6}$ & 0.33
\end{tabular}

from (10), the numerical oscillation frequency and its error relative to the theoretical electron gyro-frequency. For rotation angles up to $\theta=60^{\circ}$ the numerical electron gyro-frequency is in good agreement with the theory and the error is less than $8 \%$. For $\theta=115^{\circ}$ the computational error of electron gyro-frequency is $33 \%$ which is quite high.

\section{B. High-Resolution FDTD Magnetized Plasma Tests}

Next, the current equation solver is combined with Maxwell's equations as described in Section II, and the propagation of an electromagnetic wave inside a small plasma spherical waveguide is investigated. This test also served as a high-resolution validation test of the global FDTD plasma model of [30]. The spherical waveguide has an internal radius $2.673 \mathrm{~m}$ and external radius $3.6978 \mathrm{~m}$. A magnetic field is considered in the South-North direction and its strength is $B_{0}=0.06 \mathrm{~T}$, electron density is $n_{e}=10^{18} 1 / \mathrm{m}^{3}$. The source of the EM wave is located at $30^{\circ} \mathrm{S}$ and propagation toward Equator is examined. First, propagation of a single frequency sinusoidal wave with frequency $f=10.34 \mathrm{GHz}\left[\omega=6.5 \times 10^{10}(\mathrm{rad} / \mathrm{s})\right]$ is examined. The source creates a linearly polarized EM plane wave polarized in the $r$ direction.

According to plasma theory, only circular polarization can propagate along the magnetic field. The EM wave with linear polarization can be decomposed into a left-hand and a righthand circular polarization wave. The right-hand circular polarization wave is known as R-wave and the left-hand circular polarization wave is called L-wave. The velocity of the wave with left-hand circular polarization is different from the right-hand circular polarization wave. Because of this, the direction of polarization of the initially linearly polarized wave rotates as the wave moves along the magnetic field. This rotation is known as Faraday rotation [37]. 


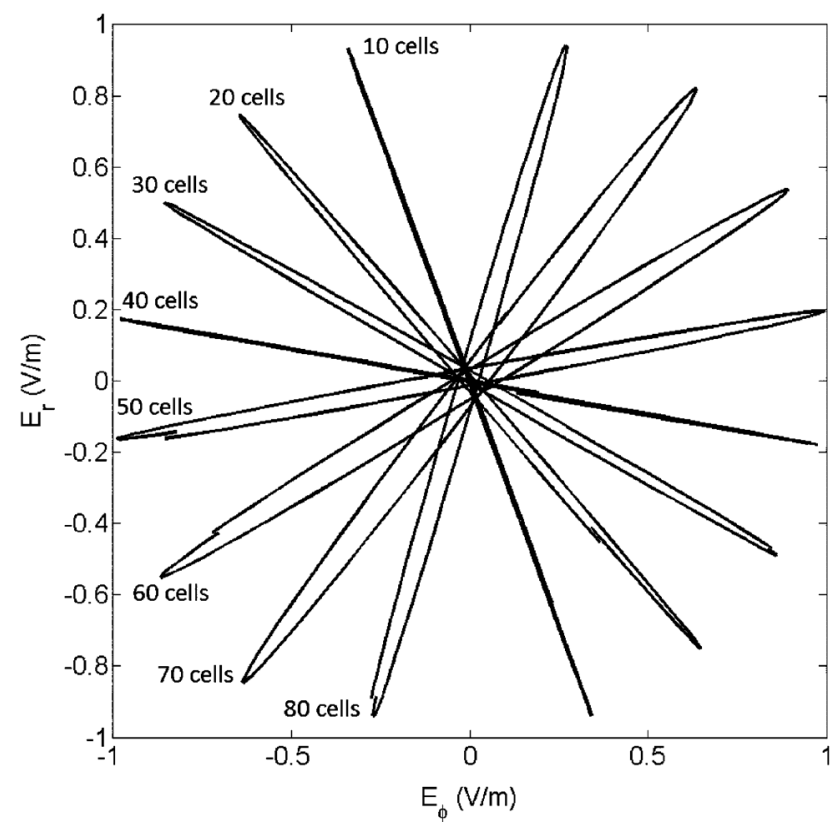

Fig. 6. Faraday rotation of an 10.34-GHz EM wave propagating along the magnetic field from $30^{\circ} \mathrm{S}$ toward the equator inside a small spherical waveguide with the internal radius $2.673 \mathrm{~m}$ and the external radius $3.6978 \mathrm{~m}$. Note that the electric field is recorded at a radius of $3.18 \mathrm{~m}$ at $10 \mathrm{~mm}, 20 \mathrm{~mm}, 30 \mathrm{~mm}, 40 \mathrm{~mm}$, $50 \mathrm{~mm}, 60 \mathrm{~mm}, 70 \mathrm{~mm}$, and $80 \mathrm{~mm}$ distances from the source and towards the Equator.

The electric field at radius $3.1858 \mathrm{~m}$ was recorded at increments of ten cells from the source towards the Equator. The resolution of the grid cells at the equator and on the internal surface of the sphere is $1 \mathrm{~mm} \times 1 \mathrm{~mm} \times 1 \mathrm{~mm}$ and on the external surface is $1 \mathrm{~mm} \times 1.3 \mathrm{~mm} \times 1.3 \mathrm{~mm}$. Fig. 6 shows the polarization of the EM wave at each observation point. The numerical Faraday rotation can be obtained from

$$
\theta_{F N}=\frac{\tan ^{-1}\left(E_{\phi} / E_{r}\right)}{d} .
$$

The error is calculated as follows:

$$
\operatorname{error}_{F}=\left(\left|\theta_{F}-\theta_{F N}\right|\right) / \theta_{F} .
$$

The error of the Faraday rotation angle is less than 1.7\%.

Using the same model, a Gaussian pulse is used for the source of the EM wave. The source electric field is described by the following expression:

$$
E_{x}=\exp \left(\frac{-(t-50 \Delta t)^{2}}{2(7 \Delta t)^{2}}\right)
$$

This pulse is expected to excite the R-wave and L-wave as well as low-frequency whistler mode. The whistler mode is part of the R-wave dispersion relation that can propagate at frequencies less than the electron gyro-frequency. Fig. 7 shows the time domain electric field in the $r$ direction, i.e., $E_{r}(t), 40$ cells (approximately $40 \mathrm{~mm}$ ) from the source. The low-frequency whistler mode arrives at the observation point at around 1.2 ps. Fig. 8 shows the frequency power spectrum of the electric field corresponding to the tie-waveform of Fig. 7. The L-wave cutoff frequency, $\omega_{L}$, the R-wave cutoff frequency, $\omega_{R}$, and the whistler mode with frequency band less than the electron cyclotron frequency $\left(\omega<\omega_{c e}\right)$ are apparent in the figure. These

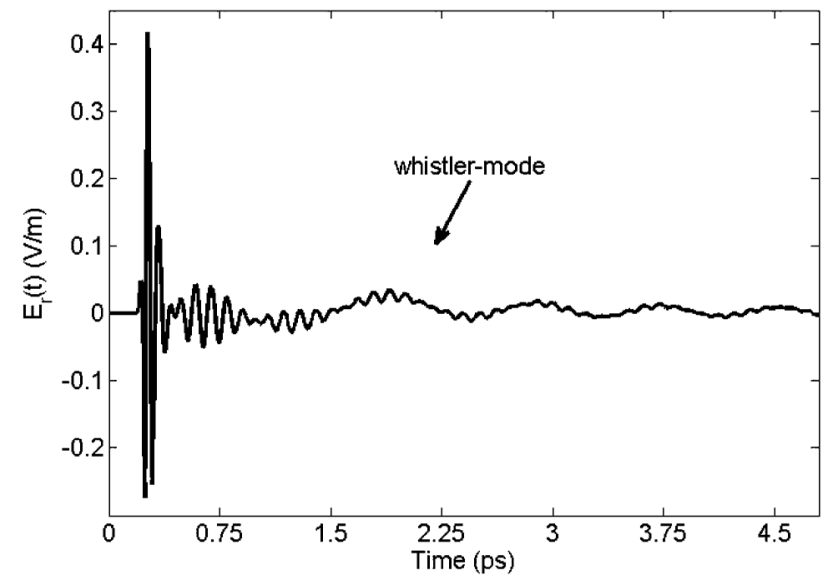

Fig. 7. Time-domain waveform of the electric field in $r$ direction recorded approximately $40 \mathrm{~mm}$ from the source along the magnetic field. The EM source is a Gaussian pulse. Approximately after 1.2 ps the whistler-mode reaches to the observation point.

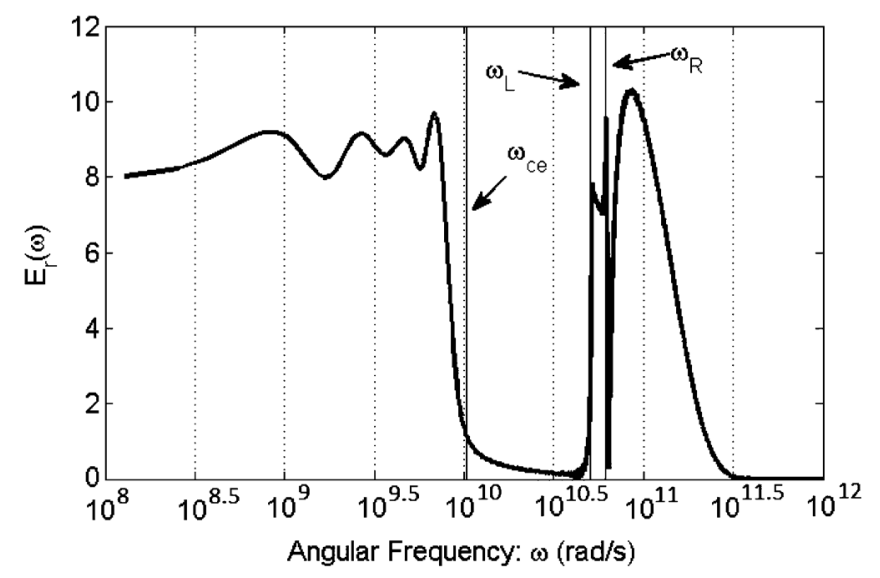

Fig. 8. Frequency power spectrum of the electric field in the $r$ direction recorded approximately $40 \mathrm{~mm}$ from the source along the magnetic field. The EM source is a Gaussian pulse described by (32). The L-wave and R-wave cutoff frequencies and whistler-mode below the electron gyro-frequency are clearly observed.

results are also in very good agreement with plasma theory and the simulation results of the previous anisotropic model [30].

Note that in these validation tests, the time step value for solving Maxwell's equation are chosen according to the Courant stability condition and is $\Delta t=1.5 \mathrm{ps}$. This time step value corresponds to a rotation angle $\theta=0.9^{\circ}$ which is much smaller than the angles of Table II. It means that the error of the numerical electron gyro-frequency is less than $0.5 \%$. Therefore, there is no need to use a different time step for solving the current equation.

\section{Global FDTD Magnetized Plasma Test}

As the final validation test, ELF propagation attenuation in the Earth-ionosphere system is investigated. This permits the use of a lower resolution global plasma model so that propagation characteristics over larger distances may be studied. This test was also performed using the isotropic and the previous anisotropic ionosphere models, both of which compared very well with previous analytical results and measurements [19], [30]. 


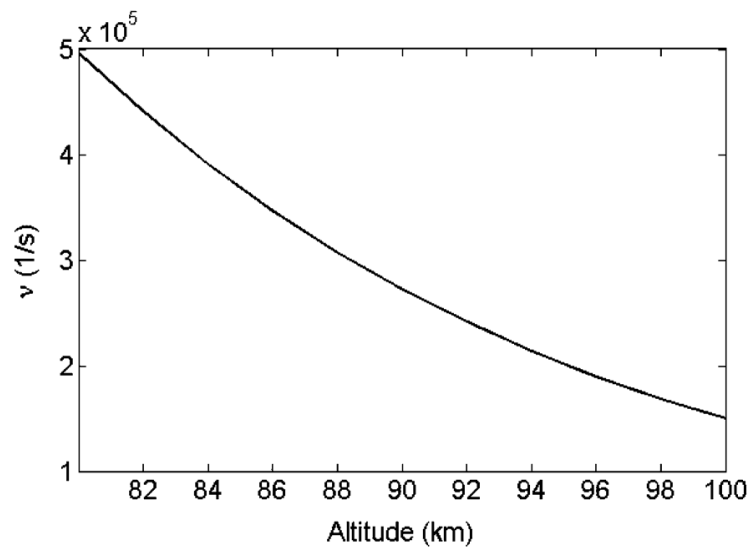

Fig. 9. Profile of the collision frequency in the ionosphere.

For this test, the magnetic field is set to the global geomagnetic field values at $100 \mathrm{~km}$ as obtained from the IGRF model. As discussed in Section II, Nyquist sampling theorem requires $\Delta t_{c}<3.57 \times 10^{-7} \mathrm{~s}$ for modeling the electron gyro-frequency without aliasing. The results of the gyro-frequency test of Table II shows that for $\Delta t_{c} \leq 1.2 \times 10^{-7} \mathrm{~s}$ the simulation results are in a good agreement with theory. The time step value for solving Maxwell's equations according to the Courant stability condition is $\Delta t=3 \times 10^{-6} \mathrm{~s}$, which is larger than the time step value required for the current equation solver. Thus, in this test, the time step value for the current equation and the Maxwell's equation are different. For the current equation solver, two time step values, $\Delta t_{c 1}=1.2 \times 10^{-7}$ $\mathrm{s}$ and $\Delta t_{c 2}=6 \times 10^{-8} \mathrm{~s}$ are chosen and the results are compared. In order to resolve the inconsistency of the time step values, for each cycle that Maxwell's equations are solved, the current density vector is updated $25\left(25 \Delta t_{c 1}=\Delta t\right)$ and $50\left(50 \Delta t_{c 2}=\Delta t\right)$ times, respectively. Note that for $\Delta t_{c 1}=1.2 \times 10^{-7} \mathrm{~s}$, two simulations are conducted. In the first, collisions are considered in the ionosphere; in the second, collisions are neglected. Fig. 9 shows the collision frequency versus altitude that is used in the collisional simulation case. The ionosphere is assumed to start from $80 \mathrm{~km}$.

Topographic and Bathymetric data are obtained from NOAA "Global Relief CD-ROM". The electron density profile and the conductivity profiles of the lithosphere and the ionosphere are similar to the previous study [30]. The current source is a 5 $\mathrm{km}$-long Gaussian pulse with a $1 / e$ full-width of $480 \Delta t$, similar to the source current used in previous studies [19]. The temporal center of the pulse is at $960 \Delta t$. The source current was above the Earth's surface at $47^{\circ} \mathrm{W}$ on the equator.

The results of the new algorithm are compared with the validated isotropic FDTD model [19]. Fig. 10 shows the attenuation of the ELF wave travelling westward from $1 / 4$ to $1 / 2$ of the distance to the antipode location for the three cases: 1) isotropic ionosphere model; 2) collisional-less anisotropic model with $\Delta t_{c 2}=6 \times 10^{-8} \mathrm{~s}$ for the current equation solver and; 3) collisional anisotropic ionosphere model with $\Delta t_{c 2}=1.2 \times 10^{-7} \mathrm{~s}$ for the current equation solver.

The ELF wave attenuations for all three cases are very similar. Simpson and Taflove [19] showed that the wave attenuation obtained from the isotropic model is in agreement with analytical predications and measurements.

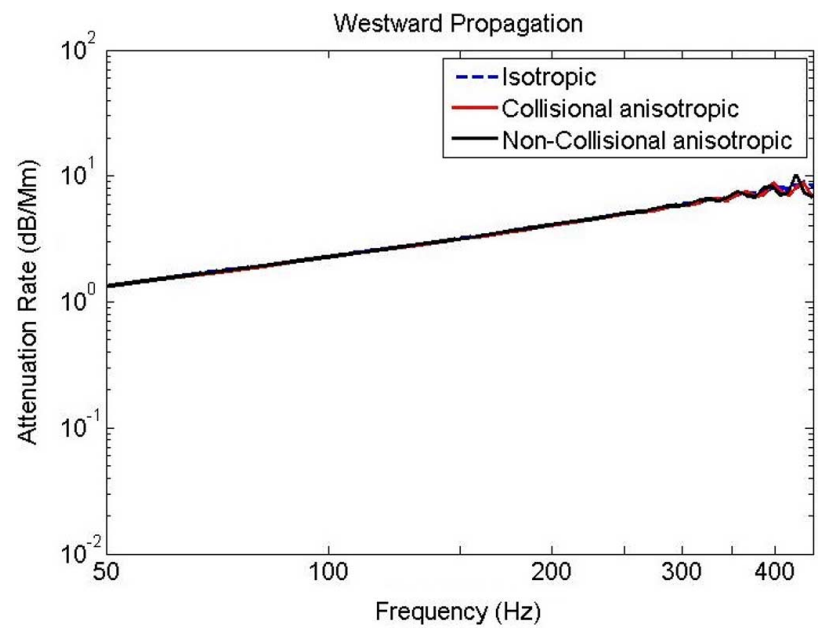

Fig. 10. ELF wave attenuation propagating westward from $1 / 4$ to $1 / 2$ of the distance from source to antipode location. Note that the dotted curve is obtained from the previous isotropic model [19].

\section{Comparison With Previous Method}

The previous anisotropic model [29], [30], solves Maxwell's Faraday equation explicitly. However, the set of the current equation and Maxwell's Ampere equation are solved implicitly. Therefore, a matrix equation must be solved to update the $\vec{E}$ fields and current density vectors $\left(\overrightarrow{J_{j}}\right)$ at each grid point (see (32) of [29]). There are three matrices $A, B$ and $C$ that are 6 $\times 6$ for only modeling electrons and $(6+3 n) \times(6+3 n)$ for modeling electrons and $n$ ion species $\left(\mathrm{O}^{+}, \mathrm{N}^{+}, \mathrm{NO}^{+}\right.$, etc.). In general the plasma frequency, gyro-frequency and collision frequency will not be constant throughout the simulation domain, so at each grid point all the components of the $A, B$ and $C$ matrices must be calculated at every time step. Then the inverse of the $A$ matrix is required and is multiplied by $B$ and $C$. Furthermore, two sets of matrices are needed for storing two time step levels of $\vec{E}$ and $\overrightarrow{J_{j}}$ : one to store their values at the current time step and the other to store the updated values. In other words, it is not possible to simply replace the updated $\vec{E}$ and $\overrightarrow{J_{j}}$ values during time-stepping.

In addition to the above complexity of implementing the previous anisotropic model [29], [30], its memory requirement limits its utility. At each Yee cell, three real numbers for the $\vec{H}$ fields, six real numbers for the coefficients of the $\vec{H}$ field equations, six real numbers for the $\vec{E}$ fields, $6(1+n)$ real numbers for the $\overrightarrow{J_{j}}$ (electrons and $n$ ion species) and $4 \times((6+3 n) \times(6+3 n))$ real numbers for the $A, A^{-1}, B$ and $C$ matrices are required.

In comparison, the method introduced in this paper solves all equations explicitly, therefore, at each Yee cell, $3+3+3+3 n$ real numbers are required for the $\vec{H}$ fields, $\vec{E}$ fields, electron current density vector, $\overrightarrow{J_{e}}$, and $n$ ion species current density vector $\overrightarrow{J_{j}}$ respectively. Six real numbers are required for the coefficients of the $\vec{H}$ field equations and six real numbers for the coefficients of the $\vec{E}$ field equations, $2(3+n)$ real numbers for $\vec{s}$ and $\vec{t}, 2(1+n)$ real numbers for electron and $n$ species ion plasma frequencies $\left(\omega_{p j}\right)$ and the corresponding collision frequencies 
$\left(\nu_{j}\right)$. Therefore, for modeling a plasma medium with electrons and $n$ ion species, at each Yee cell, the previous anisotropic model requires $21+6 n+4(6+3 n)^{2}$ real numbers in comparison to $23+7 n$ real numbers for the method introduced here.

Additionally, the maximum allowed time step value for the previous anisotropic model should meet three criteria: 1) the Courant condition [12]; 2) the Nyquist sampling condition $\Delta t<(1) /\left(2 f_{c e}\right)=(\pi) /\left(\omega_{c e}\right)$; and 3) $\nu_{j} \Delta t<0.1$. The method that is introduced here should satisfy four conditions. Two of these conditions only apply to the current equation solver: 1) the Nyquist sampling condition $\Delta t_{c}<(1) /\left(2 f_{c e}\right)=(\pi) /\left(\omega_{c e}\right)$ and 2$) \nu_{j} \Delta t_{c}<0.1$. The other two conditions apply to all equations: 1$)$ the Courant condition [12]; and 2) $\sqrt{1-\left(\omega_{p e} \Delta t / 2\right)^{2}}>0.9$. It should be mentioned again that in the new method, if $\Delta t_{c}<\Delta t$, then two different time step values can be used to solve Maxwell's equations versus the current equation. For cases in which this is not true, the ease of implementing the new algorithm along with its greatly reduced memory requirement still provide great advantages over the previous approach.

Finally, in order to provide a comparison of the execution time of the previous anisotropic model [30] and the new method, the whole Earth-ionosphere system is modeled. Both simulations are run on the same machine for only 100 time steps. The execution time of the new algorithm using $\Delta t_{c}=6 \times 10^{-8} \mathrm{~s}$ (that requires 50 iterations of the current equation solver per each time step of Maxwell's equations) was $128 \mathrm{~s}(1.28 \mathrm{~s}$ per time step) in comparison to $286 \mathrm{~s}(2.86 \mathrm{~s}$ per time step) for the previous anisotropic algorithm. Therefore, for this simulation comparison, the new algorithm is $55 \%$ faster than the previous one. A detailed comparison of the execution time between the two methods for all modeling scenarios is beyond the scope of this paper, but it is expected that the new method will always provide at least some increase in speed relative to the previous method because it avoids the matrix equations and inverse matrix calculation, and it also does not rely on as many stored numbers in the updates.

\section{CONCLUSION}

An efficient FDTD method for modeling EM wave propagation in an anisotropic magnetized ionosphere was proposed. The advantages of this model over the previous anisotropic model are:

1) It avoids having to store two levels in time both the $\vec{E}$ and $\vec{J}$ components, and it avoids having to either store or re-calculate four matrices of coefficients of size at least $6 \times 6$ at each grid cell.

2) The new algorithm is faster than the previous model. It was shown that this new algorithm is more than 50 percent faster than the previous one.

3) Implementation of this algorithm is much easier because all equations are solved explicitly and no matrix equation is required to be solved.

4) It is possible to use two different time steps for solving the current equation and Maxwell's equations. The previous anisotropic model did not have this capability and modeling the high collision frequencies was almost impossible because of the long computational time.
The proposed model was validated using five different tests ranging from high frequency localized modeling to extremely low-frequency long-distance propagation.

Propagation modeling of EM waves in the HF and microwave frequency ranges in the upper atmosphere was not feasible using the previous FDTD anisotropic models due to the overwhelming computational requirements. However, the significant improvement in execution time and less memory requirements of the new algorithm creates more possibilities for studying the propagation of higher-frequency EM waves in the upper atmosphere. Furthermore, it is more possible to extend the simulation domain to higher altitude ranges, and to more easily account for additional ion species. Inclusion of more ion species in the model makes the simulation more precise and allows us to study the physics of the wave propagation in the upper atmosphere in more detail.

\section{ACKNOWLEDGMENT}

The authors would like to thank the University of Utah's Center for High Performance Computing (CHPC) for providing supercomputing resources.

\section{REFERENCES}

[1] W. Hu and S. A. Cummer, "An FDTD model for low and high altitude lightning-generated EM fields," IEEE Trans. Antennas Propag., vol. 54, no. 5, pp. 1513-1522, May 2006.

[2] A. V. Gurevich, "Nonlinear effects in the ionosphere," Phys.-Usp., vol. 50, 2007 [Online]. Available: doi:10.1070/ PU2007v050n11ABEH006212

[3] M. R. Bordikar, W. A. Scales, A. Samimi, P. A. Bernhardt, S. Briczinski, and M. J. McCarrick, "First observations of minority ion $(\mathrm{H}+)$ structuring in stimulated radiation during second electron gyro-harmonic heating experiments," Geophys. Res. Lett., vol. 40, pp. 1479-1483, 2013.

[4] M. R. Bordikar, W. A. Scales, A. Mahmoudian, H. Kim, P. A. Bernhardt, R. Redmon, A. Samimi, S. Brizcinski, and M. J. McCarrick, "Impact of active geomagnetic conditions on stimulated radiation during ionospheric second electron gyro-harmonic heating," J. Geophys. Res. Space Phys., vol. 119, no. 1, pp. 548-565, Jan. 2014.

[5] T. B. Leyser, "Stimulated electromagnetic emission by high-frequency electromagnetic pumping of the ionospheric plasma," Space Sci. Rev., vol. 98 , pp. 223-328, 2001.

[6] W. A. Scales, M. R. Bordikar, A. Samimi, P. A. Bernhardt, S. Briczinski, C. A. Selcher, and M. McCarrick, "Observations and theory of ion gyro-harmonic structures in the stimulated radiation spectrum during second electron gyro-harmonic heating," presented at the URSI General Assembly and Scientific Symp., 2011.

[7] A. Samimi, W. A. Scales, P. A. Bernhardt, S. J. Briczinski, C. A. Selcher, and M. J. McCarrick, "On ion gyro-harmonic structuring in the stimulated electromagnetic emission spectrum during second electron gyro-harmonic heating," Ann. Geophys., vol. 30, pp. 1587-1594, 2012.

[8] A. Samimi, W. A. Scales, H. Fu, P. A. Bernhardt, S. J. Briczinski, and M. J. McCarrick, "Ion gyroharmonic structures in stimulated radiation during second electron gyroharmonic heating: 1. Theory," J. Geophys. Res: Space Phys., vol. 118, pp. 502-514, 2013.

[9] H. Fu, W. A. Scales, P. A. Bernhardt, A. Samimi, A. Mahmoudian, S. J. Briczinski, and M. McCarrick, "Stimulated Brillouin scatter and stimulated ion Bernstein scatter during electron gyro-harmonic heating experiments," Radio Sci., vol. 48, pp. 607-616, 2013.

[10] A. Mahmoudian, W. A. Scales, P. A. Bernhardt, A. Samimi, E. Kendall, J. M. Ruohoniemi, B. Isham, and M. Bordikar, "Ion gyro-harmonic structuring in the stimulated radiation spectrum and optical emissions during electron gyro-harmonic heating," J. Geophys. Res., Space Phys., vol. 118, pp. 1270-1287, 2013.

[11] A. Samimi, W. A. Scales, P. A. Bernhardt, S. J. Briczinski, and M. J. McCarrick, "Ion gyroharmonic structures in stimulated radiation during second electron gyroharmonic heating: 2. Simulations," $J$. Geophys. Res. Space Phys., vol. 119, pp. 462-478, 2014. 
[12] A. Taflove and S. C. Hagness, Computational Electromagnetics: $\mathrm{Fi}$ nite-Difference Time-Domain Method. Norwell, MA, USA: Artech House, 2005.

[13] M. Thvenot, J. P. Brenger, T. Monedire, and F. Jecko, "A FDTD scheme for the computation of VLF-LF propagation in the anisotropic earth-ionosphere waveguide," Ann. Telecomm., vol. 54, pp. 297-310, 1999.

[14] J. P. Berenger, "FDTD computation of VLF-LF propagation in the Earth-ionosphere waveguide," Ann. Telecomm., vol. 57, no. 11-12, pp. 1059-1090, 2002.

[15] S. A. Cummer, "Modeling electromagnetic propagation in the Earthionosphere waveguide," IEEE Trans. Antennas Propag., vol. 48, no. 9, pp. 1420-1429, Sep. 2000.

[16] Y. Todoroki, S. Maekawa, T. Yamauchi, T. Horie, and M. Hayakawa, "Solar flare induced D region perturbation in the ionosphere, as revealed from a short-distance VLF propagation path," Geophys. Res. Lett., vol. 34, no. L03103, 2007.

[17] Y. T. Tanaka, T. Terasawa, M. Yoshida, T. Horie, and M. Hayakawa, "Ionospheric disturbances caused by SGR giant gamma ray flare in 1998: Constraints on the energy spectrum of the flare," J. Geophys. Res., vol. 113, no. A07307, 2008.

[18] M. Hayakawa and T. Otsuyama, "FDTD analysis of ELF wave propagation in inhomogeneous sub ionospheric waveguide models," Appl. Comput. Electromagn. Soc. J., vol. 17, no. 3, pp. 239-244, 2002.

[19] J. J. Simpson and A. Taflove, "Three-dimensional FDTD modeling of impulsive ELF antipodal propagation and Schumann resonance of the Earth-sphere," IEEE Trans. Antennas Propag., vol. 52, no. 2, pp. 443-451, Feb. 2004.

[20] H. Yang and V. P. Pasko, "Three-dimensional finite-difference timedomain modeling of the Earth-ionosphere cavity resonances," Geophys. Res. Lett., vol. 32, no. L03114, 2005.

[21] J. J. Simpson and A. Taflove, "A review of progress in FDTD Maxwells equations modeling of impulsive sub-ionospheric propagation below 300 kHz," IEEE Trans. Antennas Propag., vol. 55, no. 6, pp. 1582-1590, Jun. 2007.

[22] J. J. Simpson, "Current and future applications of full-vector 3-D Maxwell's equations FDTD global earth-ionosphere waveguide models," Surv. Geophys., vol. 30, no. 2, pp. 105-130, 2009.

[23] P. Bannister, "ELF propagation update," IEEE J. Ocean. Eng., vol. 0E-9, no. 3, pp. 179-188, 1984.

[24] J. J. Simpson and A. Taflove, "ELF radar system proposed for localized D-region ionospheric anomalies," IEEE Geosci. Remote Sens. Lett., vol. 3, no. 4, pp. 500-503, Oct. 2006.

[25] J. J. Simpson and A. Taflove, "A novel ELF radar for major oil deposits," IEEE Geosci. Remote Sens. Lett., vol. 3, no. 1, pp. 36-39, Jan. 2006.

[26] J. J. Simpson, R. P. Heikes, and A. Taflove, "FDTD modeling of a novel ELF radar for major oil deposits using a three-dimensional geodesic grid of the Earth-ionosphere waveguide," IEEE Trans. Antennas Propag., vol. 54, no. 6, pp. 1734-1741, Jun. 2006.

[27] J. J. Simpson and A. Taflove, "Electrokinetic effect of the Loma Prieta earthquake calculated by an entire-Earth FDTD solution of Maxwell's equations," Geophys. Res. Lett., vol. 32, no. L09302, 2005.

[28] J. J. Simpson, "On the possibility of high-level transient coronal mass ejection-induced ionospheric current coupling to electric power grids," J. Geophys. Res.-Space Phys., vol. 116, no. A11308, 2011.

[29] Y. Yu and J. J. Simpson, "An E-J collocated 3-D FDTD model of electromagnetic wave propagation in magnetized cold plasma," IEEE Trans. Antennas Propag., vol. 58, no. 2, pp. 469-478, Feb. 2010.

[30] Y. Yu, J. Niu, and J. J. Simpson, “A 3-D global Earth-ionosphere FDTD model including an anisotropic magnetized plasma ionosphere," IEEE Trans. Antennas Propag., vol. 60, no. 7, pp. 3246-3256, Jul. 2012.
[31] J. P. Bérenger, "An implicit FDTD scheme for the propagation of VLFLF radio waves in the Earth-ionosphere waveguide," Comptes Rendus Phys., vol. 15, no. 5, pp. 393-402, 2014.

[32] J. P. Boris, "The acceleration calculation from a scalar potential," Plasma Phys. Lab., Princeton Univ., MATT-152, Mar. 1970.

[33] C. K. Birdsall and A. B. Langdon, Plasma Physics Via Computer Simulation. New York, NY, USA: Inst. of Phys., 1991.

[34] A. V. Oppenheim and R. W. Schafer, Discrete-Time Signal Processing, 3rd ed. Upper Saddle River, NJ, USA: Prentice-Hall, 2009, Signal Processing.

[35] G. A. Sod, "A survey of several finite difference methods for systems of nonlinear hyperbolic conservation laws," J. Comp. Phys., vol. 27, no. 1, pp. 1-31, 1978.

[36] R. Garcia and R. A. Kahawita, "Numerical solution of the St. Venant equations with the MacCormack finite-difference scheme," Int. J. Numer. Meth. Fluids, vol. 6, pp. 259-274, 1986.

[37] F. F. Chen, Introduction to Plasma Physics and Controlled Fusion, 2nd ed. New York, NY, USA: Springer, 1984, Plasma Physics.

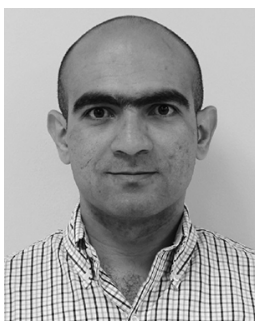

Alireza Samimi (S'11-M'13) received the B.S. degree in electrical engineering from Shiraz University, Shiraz, Iran, in 2005, the M.S. degree in electrical engineering from University of Tabriz, Tabriz, Iran, in 2008, and the Ph.D. degree in electrical engineering from Virginia Polytechnic Institute and State University, Blacksburg, VA, USA, in 2013.

His research interests include physics of the upper atmosphere, finite-difference time-domain (FDTD) solution of Maxwell's equations and its applications in simulating wave propagation in the Earth-ionosphere-magnetosphere system, active modification of the ionosphere, and particle-in-cell computational modeling of plasma instabilities. He is currently a Postdoctoral Fellow in the Department of Electrical and Computer Engineering, University of Utah, Salt Lake City, UT, USA.

Dr. Samimi has been a member of the American Geophysical Union (AGU) since 2010 .

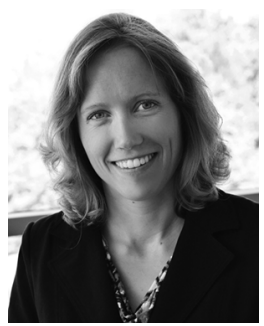

Jamesina J. Simpson (S'01-M'07-SM'12) received the B.S. and Ph.D. degrees in electrical engineering from Northwestern University, Evanston, IL, USA, in 2003 and 2007, respectively.

In 2007, she joined the Electrical and Computer Engineering Department, University of New Mexico, Albuquerque, NM, USA, as a tenure-track Assistant Professor. As of July 2012, she has been an Associate Professor in the Electrical and Computer Engineering Department, University of Utah, Salt Lake City, UT, USA. Her research lab encompasses the application of the full-vector Maxwell's equations finite-difference time-domain (FDTD) method to a wide variety of scientific and engineering applications across the electromagnetic spectrum.

Dr. Simpson is a recipient of the National Science Foundation (NSF) Graduate Research Fellowship and the IEEE AP-S and MTT-S Graduate Research Awards. In 2010, she received an NSF CAREER Award and in 2012 she received the Donald G. Dudley, Jr. Undergraduate Teaching Award of the IEEE AP-S. From 2010 to 2014, she was an Associate Editor of the IEEE TRANSACTIONS ON ANTENNAS AND PROPAGATION. 